
S. $\operatorname{AGAN}^{1}$
F. $A Y^{2}$
A. KOCABAS ${ }^{2}$
A. AYDINLI ${ }^{2, \square}$

\title{
Stress effects in prism coupling measurements of thin polymer films
}

\author{
${ }^{1}$ Department of Physics, Kirikkale University, 71450 Kirikkale, Turkey \\ ${ }^{2}$ Department of Physics, Bilkent University, 06800 Ankara, Turkey
}

\section{Received: 5 March 2003/Accepted: 10 May 2003 \\ Published online: 8 July 2003 • (C) Springer-Verlag 2003}

ABSTRACT Due to the increasingly important role of some polymers in optical waveguide technologies, precise measurement of their optical properties has become important. Typically, prism coupling to slab waveguides made of materials of interest is used to measure the relevant optical parameters. However, such measurements are often complicated by the softness of the polymer films when stress is applied to the prism to couple light into the waveguides. In this work, we have investigated the optical properties of three different polymers, polystyrene (PS), polymethyl-methacrylate (PMMA), and benzocyclobutane (BCB). For the first time, the dependence of the refractive index, film thickness, and birefringence on applied stress in these thin polymer films was determined by means of the prism coupling technique. Both symmetric trapezoid shaped and right-angle prisms were used to couple the light into the waveguides. It was found that trapezoid shaped prism coupling gives better results in these thin polymer films. The refractive index of PMMA was found to be in the range of 1.4869 up to 1.4876 for both TE and TM polarizations under the applied force, which causes a small decrease in the film thickness of up to $0.06 \mu \mathrm{m}$. PMMA waveguide films were found not to be birefringent. In contrast, both BCB and PS films exhibit birefringence albeit of opposing signs.

PACS 42.82.Et; 78.20.Ci; 78.20.Fm

\section{1}

\section{Introduction}

A growing number of investigations are currently being carried out on polymeric amorphous materials, because of their high figure of merit for photonic applications [1-4]. The optical properties of polymer films are of great importance for optical components and sensor applications as well as in a host of opto-electronic devices and in magneto-optical recording $[5,6]$. While many kinds of polymers can be used in integrated optics (IO) devices and in the microelectronics industry, polymethyl-methacrylate (PMMA), benzocyclobutane (BCB), and polystyrene (PS) are among the most promising $[7,8]$. Polymers are typically spin coated onto substrates where the spin-coating and curing processes may introduce

Fax: +90-312/266-45-79, E-mail: aydinli@fen.bilkent.edu.tr in-plane orientation of polymeric chains. It is proposed that this is due to a biaxial tensile stress caused by substrate confinement when the solvent evaporates [9]. This leads to a molecular ordering, resulting in in-plane and out-of-plane optical anisotropy for the thin polymer films, as was confirmed by several investigations analyzing optical anisotropy for films with thicknesses in the few-micrometers range $[10,11]$.

Development of optical waveguide techniques has provided a convenient method for measuring the refractive indices and thicknesses of thin dielectric films. Due to the ease of operation and high measurement accuracy, the prism waveguide coupler has been used to determine the refractive index and birefringence of polymer films as well [12-15]. It is important to characterize thin polymer films since their properties in thin-film form may differ by several orders of magnitude from those of bulk polymers [16]. The prism waveguide coupler is particularly suitable for isotropic and anisotropic polymer thin-film studies because of the quantitative character of the information obtained.

To study optical and mechanical properties of polymeric systems, birefringence measurements have been used for a long time $[17,18]$. The most important origin of birefringence in polymeric thin films is the chain orientation of molecules comprising the polymer, which depends on the method of preparation of the thin film. As discussed above, intrinsic stress during sample preparation is also related to the chain orientation. Finally, extrinsic stress applied during the measurement process may influence the observed stress considerably.

2

\section{Experimental}

A schematic representation of the prism coupling measurement (PCM) setup which was built in our laboratory is given in Fig. 1.

Alignment optics includes a polarization rotator and an analyzer in addition to a chopper. A beam splitter was also included to calibrate the relative orientation of the laser beam with respect to the coupling prism and was used to establish the origin of the angular displacement. The coupling pressure was adjusted by a micrometer holder in contact with a calibrated spring system that allowed us to monitor the force applied to the prism. The operational procedure of the PCM is simple. The linearly polarized monochromatic light, 


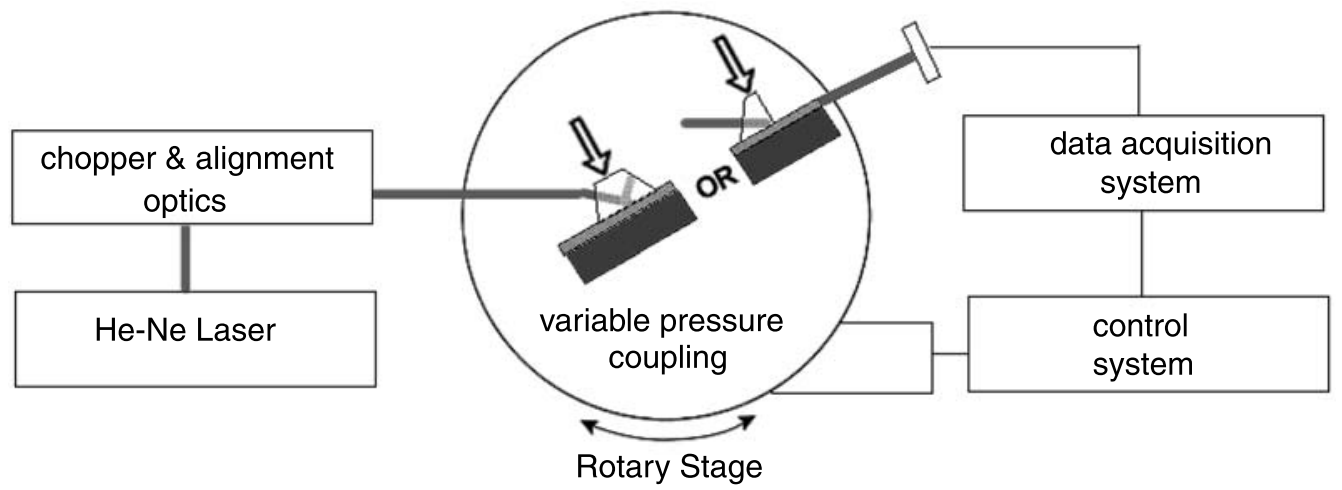

FIGURE 1 Experimental setup for measuring the coupling angles. The laser beam is incident on the coupling prism. The prism coupler setup is mounted on a high-precision rotary stage with stepper motors with a precision of better than $\pm 0.01^{\circ}$

of 632.8-nm wavelength from a He-Ne laser, with either transverse electric (TE) or transverse magnetic (TM) polarization is incident onto the prism. The waveguide and the coupling prism are rotated on a high-precision motorized rotary stage on which they are mounted under computer control. All the measurements were performed by using a single prism to determine the coupling angles of each mode. Initially, both symmetric trapezoid shape and right-angle prisms made of SF-14 with base angles of $60^{\circ}$ and refractive index of 1.7561 $(\lambda=632.8 \mathrm{~nm})$ were tried. Guided intensity is measured on the opposite side of the waveguide by a $\mathrm{Si}$ photodetector as a function of the incident angle. To minimize the noise, we used a lock-in amplifier which was connected to a computer that also controlled the rotary motor. From the angles at which local intensity maxima are observed, the refractive index and thickness of the measured film can be obtained by solving the waveguide mode equations for TE and TM polarizations $[1,16,19]$. Attention must be paid towards properly aligning the coupling prism. The repeatability of the measured coupling angle has been checked and found to be less than $\pm 0.01^{\circ}$. The intensity analysis method established in this study has been applied to obtain the refractive indices of the PMMA, BCB, and PS thin polymer films. The typical error in the refractive-index and thickness values is found to be less than $\pm 0.0002 \%$ and $\pm 0.3 \%$, respectively. The measurements were all performed during the unloading process. All the experiments were carried out at a constant temperature of $21.0 \pm 1.0^{\circ} \mathrm{C}$.

To prepare optical waveguide structures for prism coupling (PC) measurements, fabricating thin films is an important first step. PMMA, BCB, and PS are all well suited for fabricating excellent polymer waveguide layers by means of spin coating [1]. In this work, the PMMA and PS were obtained from Sigma-Aldrich. The molecular weights (Mws) of PMMA and PS were 15000 and 150000 , respectively. The PMMA and PS polymers were dissolved in chloroform at $15.0 \mathrm{wt} . \%$ and $6.0 \mathrm{wt} . \%$, respectively. The polymer solution obtained in this manner was prepared by spin coating from the solution at 3000 and $2000 \mathrm{rpm}$ for $40 \mathrm{~s}$ on a $\mathrm{SiO}_{2}$ coated $\mathrm{Si}$ wafer. The films were then cured at a temperature of $110^{\circ} \mathrm{C}$ for $30 \mathrm{~min}$. Benzocyclobutane (BCB) thin films were spin coated at spin speeds of $5000 \mathrm{rpm}$ for $40 \mathrm{~s}$. Fine control of the film thickness was best obtained by adjusting the spin speed. The BCB polymer is supplied as a metasilyene solution of the pre-polymer. The pre-polymer cyclotone 3022-46 was obtained from Dow Chemical Company for this purpose.
These samples were cured in an oven under nitrogen atmosphere at $250{ }^{\circ} \mathrm{C}$ for $60 \mathrm{~min}$. The substrates were silicon slices of $3 \mathrm{~cm} \times 1.5 \mathrm{~cm}$ with $\mathrm{CVD}$-grown $\mathrm{SiO}_{2}$ layers. The thickness of the $\mathrm{SiO}_{2}$ layers was $7.2 \mu \mathrm{m}$ and the refractive index was 1.4568 at $632.8 \mathrm{~nm}$. PMMA, PS, and BCB layers had thicknesses of $2.30,2.37$, and $2.38 \mu \mathrm{m}$, as measured by a stylus profilometer (Sloan Dektak 3030ST), respectively.

\section{$3 \quad$ Results and discussion}

The polymer waveguides were characterized for their refractive index, film thickness, and birefringence. To measure the dependence on the applied force of the refractive indices and film thicknesses, we used the prism coupling method (PCM). In the experiments, two types of prisms were used: a symmetric trapezoid prism (Fig. 2b) and a right-angle prism (Fig. 2a). An example of the coupled intensity as a function of rotation angle for both prisms is shown in Fig. 2.

As is clearly observed in Fig. 2a, the right-angle prism gives broad peaks that are shifted with respect to those obtained by the symmetric trapezoid prism. The broadening of the peaks results in lower precision in the exact determination of the mode-coupling angle, leading to larger errors in the refractive index and film thickness. The shift of the mode-coupling angle results in loss of accuracy in de-

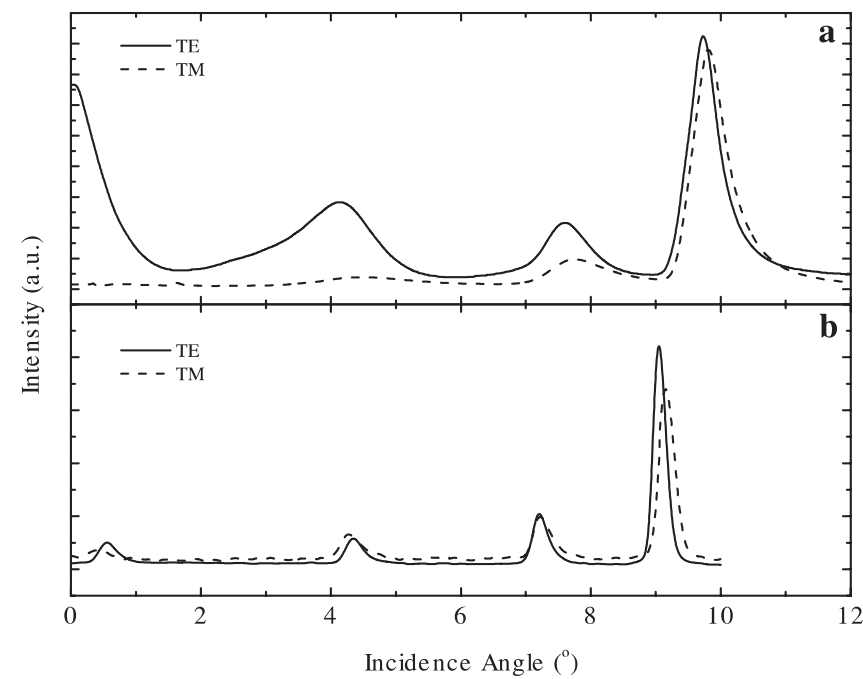

FIGURE 2 Coupled light spectrum for a triangle- and b trapezoid-shaped prisms for PS films 
termination of the mode-coupling angle and hence in the refractive index and film thickness. The application of loading force on the right-angle prism results in the rotation of the prism about an axis through the coupling edge during the measurement, due to the softness of the underlying polymer film. Coupled with a local reduction of thickness under the prism in the coupling region, this leads to both broadening and shift of the observed peaks. Repeated measurements show that a coupling spectrum with narrower coupling peaks is obtained using a symmetric trapezoid prism. A symmetric trapezoid prism was used throughout the rest of these experiments. The results showed that symmetric trapezoid prisms are more suitable for measurement of refractive index and thickness using PC methods in thin-film polymer waveguides.

With the introduction of the prism coupling method by Tien and Ulrich the possibility of the coupling strength causing a shift and broadening of the modes due to presence of the prism in the vicinity of the film has been argued $[13,15]$. Decrease of the air gap that originates from the dust particles and is located between the prism and the film due to the applied pressure has been seen as the source of this artifact. Recent theoretical analysis made to estimate the angular shift observed in the $m$-line prism coupling method by Monneret et al. [20] shows that for a $\mathrm{SrTiO}_{3}$ prism and a film with a refractive index of 2.27, the shift results in an index change of $5 \times 10^{-5}$, which is well below our precision. In our experiments, the prism used is of lower refractive index and the index contrast between the film and the prism is low, which should result in even smaller changes in the refractive index [13]. Additionally, the decrease of the air gap and thus the coupling efficiency is a function of the mechanical properties of the film under investigation. Our thin-film polymers are softer than the ordinary glassy dielectric materials [21]. Therefore, it is reasonable to assume that decreasing of the air-gap thickness with applied pressure is small in the range of the thickness variations of the polymer films used in this work. Figure 3 shows the coupling intensity dependence on the applied force.

It can be seen from the figure that the pressure is critical in getting optimum coupling efficiency. When the air gap between prism and polymer film is about a fraction of $\lambda$, maximum energy is transferred into the polymer waveguide [22]. However, it should be noted that, as long as there is a sufficient signal to noise ratio, accurate measurements of the coupling angles can be made under a variety of applied stresses.

Considering that the polymer films form a slab waveguide with $\mathrm{SiO}_{2}$ as the lower cladding and air acting as the upper cladding, the number of modes for both TE and TM polarizations were calculated by solving Maxwell's equations with the corresponding boundary conditions [23]. Finally, the number of modes was also confirmed with the calculations of the mode spectrum using beam-propagation simulations employing the finite-difference approach. The number of modes calculated in this manner was in agreement with the number of modes observed in these measurements. In Fig. 4, the vertical line represents the out-coupled mode spectrum excited for $m=0,1$, and 2 modes for TE polarization and $m=0$ and 1 modes for TM polarization. Two guided modes have been excited with $\mathrm{TM}$ polarization at $-2.475^{\circ}$ and $-3.825^{\circ}$ angles, while three modes have been obtained in the TE spectrum at $-2.490^{\circ},-3.885^{\circ}$, and $-5.230^{\circ}$.

Starting from the angles of TE and TM (in Fig. 4) guided modes, both the refractive index and thickness of our waveguide polymer films were computed.

Due to the softness of the polymer films, a reduction in polymer film thickness may be expected as the applied stress is increased, as indeed is observed experimentally. A change in refractive index is also observed, which is shown in Fig. 5 as a function of the reduction in thickness of the polymer film for PMMA samples.

As can be seen from Fig. 5, the refractive index of a PMMA thin film increases with increasing applied stress. The dependence of refractive index on applied stress is classically explained by the Neumann-Maxwell equations.

$n_{x}=n_{0 x}+C_{1} \sigma_{x}+C_{2}\left(\sigma_{y}+\sigma_{z}\right)$,
$n_{y}=n_{0 y}+C_{1} \sigma_{y}+C_{2}\left(\sigma_{x}+\sigma_{z}\right)$,

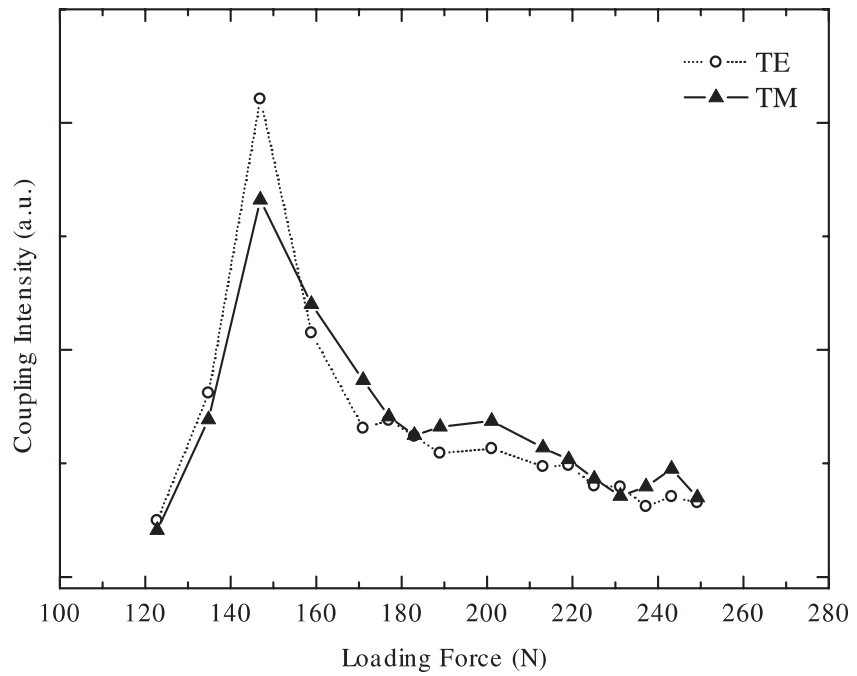

FIGURE 3 Light coupling efficiency vs. loading force for both TE and TM polarizations as observed for PS films

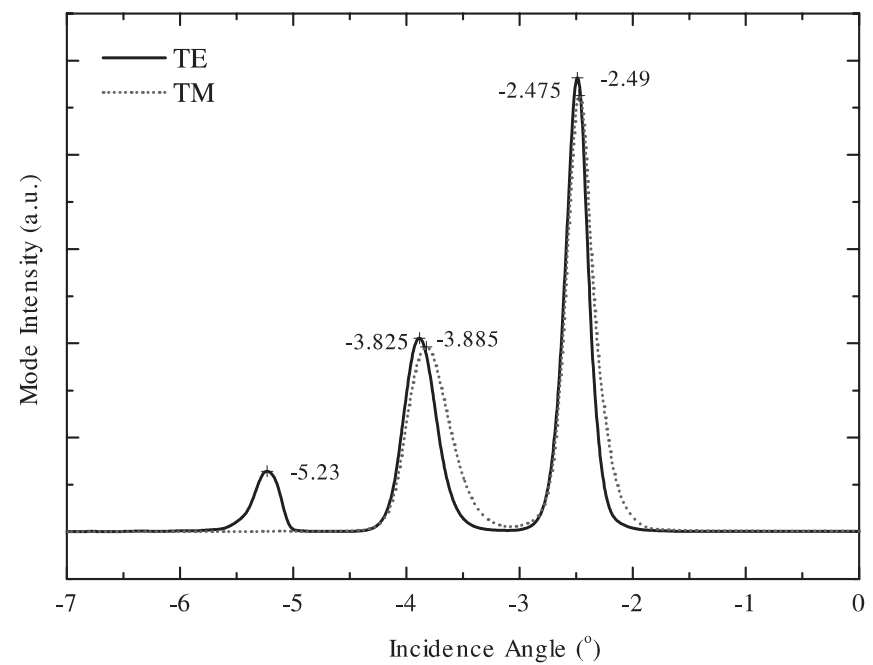

FIGURE 4 Typical spectrum of guided modes with TM (or TE) polarized light for PMMA 


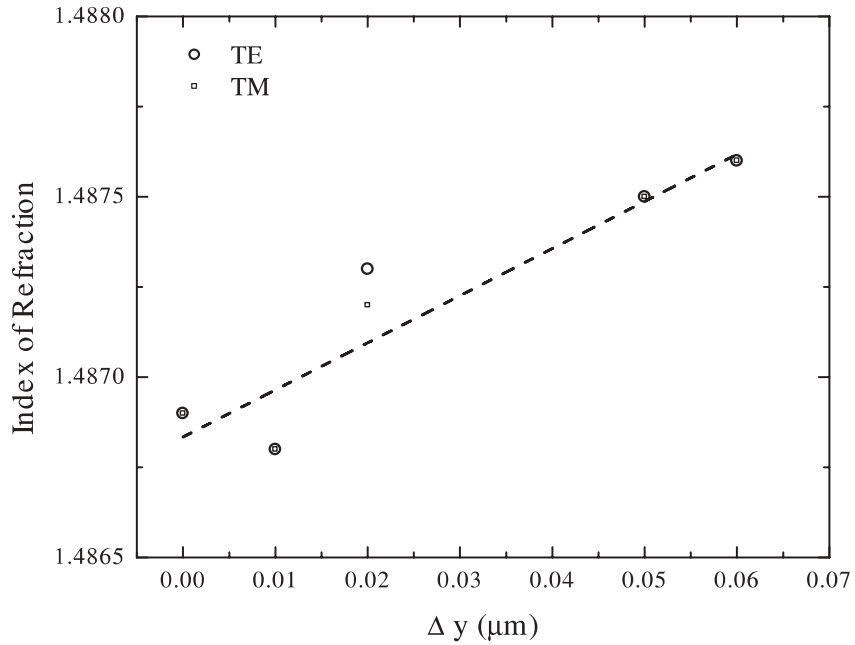

FIGURE 5 Calculated refractive-index values vs, thickness change of PMMA films. A small but steady increase of the refractive index is clearly observed for both polarizations

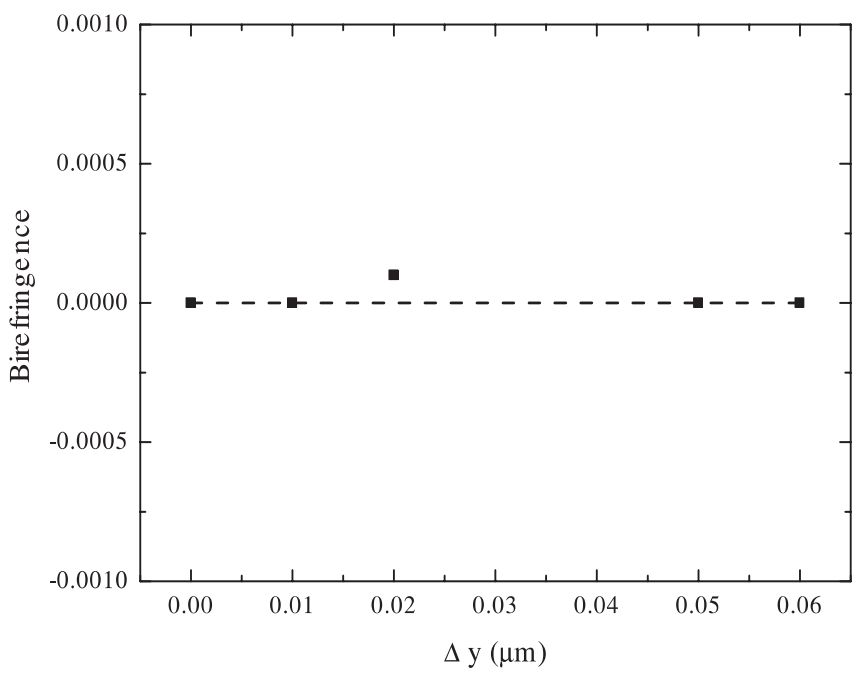

FIGURE 6 Birefringence vs. thickness change of PMMA polymer films

where $n_{x}, n_{y}$ is the refractive index experienced by a light wave polarized in the direction of a principal axis (i.e. $x \sim \mathrm{TE}$, in-plane and $y \sim \mathrm{TM}$, out-of-plane polarizations). $n_{0 x}$ and $n_{0 y}$ are refractive indices of the unstressed material, $C_{1}$ and $C_{2}$ are stress-optic coefficients, and $\sigma_{x}, \sigma_{y}$, and $\sigma_{z}$ are applied stresses for in-plane, out-of-plane, and light-propagation axes, respectively [17]. The applied stresses in all directions are not known for the calculation of the stress dependence of the refractive indices, in our case. Therefore, we have used the finiteelement method (FEM) to calculate the missing stress components to overcome this problem. The details of extrinsic stress and its effects on the opto-mechanical properties of our polymer waveguides and optical properties of PMMA, PS, and
BCB polymer films are discussed in detail elsewhere [24]. Applied stress causes a steady decrease of film thickness, resulting in a displacement reduction of up to $0.06 \mu \mathrm{m}$. The value of the refractive index extrapolated to zero applied stress is found to be 1.4869. As the refractive index of bulk PMMA is given to be between 1.48 and 1.49 [25], the value we obtained is in very good agreement with the literature. At higher stress values represented with the film-thickness displacement in Fig. 5, refractive index increases at a rate of $0.0133 \mu \mathrm{m}^{-1}$ in the elastic region for both TE and TM polarizations.

A nonzero value of $\Delta n=n_{\mathrm{TE}}-n_{\mathrm{TM}}$ indicates that the material is birefringent and hence anisotropic. Birefringence gives the level of optical anisotropy in the film, which is the difference in refractive index between orthogonal planes of polarization. Thus, birefringence is a measure of the molecular orientation. The data in Fig. 6 reflect the birefringence of PMMA.

Birefringence of PMMA slab waveguides was found to be zero. This result is supported in the literature [25]. Additionally, negative birefringence was observed for PS and positive birefringence for BCB slab waveguides with values of $\Delta n=-0.0008$ and $\Delta n=+0.0022$ at the unstressed condition $(\Delta y=0 \mu \mathrm{m})$, respectively. The birefringence results indicate that for $\mathrm{BCB}$ and PS films the polymer molecules are preferentially oriented in and out of the film plane, respectively [8]. An increase of the applied pressure on the prism, accompanied by the film-thickness reduction $(\Delta y)$ results in enhancement of the negative birefringence of PS and decrease of the positive birefringence of $\mathrm{BCB}$ films. Both can be attributed to the greater increase of $n_{\mathrm{TM}}$ compared to $n_{\mathrm{TE}}$ as the stress is increased in the out-of-plane direction for both PS and BCB layers, understood in the framework of the Neumann-Maxwell equations (1). Table 1 summarizes the results of optical properties of PMMA, PS, and BCB polymer thin films.

\section{4}

\section{Conclusions}

We have measured the refractive index and thickness of spin-coated polymers on silicon substrates by using the well-known prism coupling technique. It has become clear during our measurements that symmetric trapezoid prisms are better suited to couple light into the polymer thin film instead of right-angle prisms. Applied stress results in the elastic reduction of the polymer thin-film thickness as well as an increase in the refractive index. Measurements of the refractive index for both TE and TM polarizations for PMMA, BCB, and PS thin films all show that refractive index increases with applied stress, which is understood within the framework of the Neumann-Maxwell equations. Making use of finite-element calculations of unknown components of stress, we were able to determine the birefringence of all three polymer films as

\begin{tabular}{lllllllll}
\hline & & \multicolumn{3}{c}{$\Delta \mathrm{y}=0 \mu \mathrm{m}$} & \multicolumn{2}{c}{$\Delta \mathrm{y}=\Delta \mathrm{y}_{\max }$} \\
& $n_{0 \mathrm{TE}}$ & $n_{0 \mathrm{TM}}$ & $t_{0}(\mu \mathrm{m})$ & $n_{0 \mathrm{TE}}-n_{0 \mathrm{TM}}$ & $n_{\mathrm{TE}}$ & $n_{\mathrm{TM}}$ & $t(\mu \mathrm{m})$ & $n_{\mathrm{TE}}-n_{\mathrm{TM}}$ \\
\hline PMMA & 1.4869 & 1.4869 & 2.30 & 0 & 1.4876 & 1.4876 & 2.24 & 0 \\
PS & 1.5844 & 1.5852 & 2.37 & -0.0008 & 1.5857 & 1.5874 & 2.21 & -0.0017 \\
BCB & 1.5575 & 1.5553 & 2.38 & +0.0022 & 1.5586 & 1.5570 & 2.31 & +0.0016 \\
\hline
\end{tabular}

TABLE 1 Summary of all refractive-index and thickness measurements 
a function of applied stress. We have shown for the first time that, in contrast with PMMA thin films which showed no birefringence at any applied stress levels used in this study, PS and $\mathrm{BCB}$ both showed increasing birefringence as a function of applied stress, the former of negative sign and the latter of positive sign. We have shown that the prism coupling method is a useful technique to measure opto-mechanical properties of thin polymer films, with proper choice of the coupling prism and use of finite-element methods to calculate unknown stress components.

ACKNOWLEDGEMENTS We wish to thank Prof. Soner Kilic (Bilkent University) and Prof. Levent Toppare (Middle East Technical University) for their comments on the preparation of the polymer films. We would also like to thank First Lt. Fatma Donmez (Optics Department of 1010th Ordnance Main Depot) for supplying the prisms used in this study. One of us (S.A.) thanks Bilkent University Physics Department for the hospitality shown during his stay. This work was supported, in part, by Bilkent University Research Fund (Code: Phys-03-02) and the Scientific and Technical Research Council of Turkey (TUBITAK, Project No. 199E006).

\section{REFERENCES}

1 F. Michelotti, T. Gabler, H. Horhold, R. Waldhousl, A. Brauer: Opt. Commun. 114, 247 (1995)

2 K. Sasaki, K. Fujii, T. Tornioka, T. Kinoshita: J. Opt. Soc. Am. B 5, 457 (1988)
3 R. Burzyanski, B.P. Singh, P.N. Prasad, R. Zanoni, G.I. Stegeman: Appl. Phys. Lett. 53, 2011 (1988)

4 C.B. Rider, J.S. Schilckraut, M. Scozzafava: J. Appl. Phys. 70, 29 (1991)

5 A.M. Nasr: Polym. Test. 21, 303 (2002)

6 K. Fischer, J. Muller: Sens. Actuators, B 9, 209 (1992)

7 G. Arun, V.K. Sharma, A. Kapoor, K.N. Tripathi: Opt. Laser Technol. 34, 395 (2002)

8 M. Ree, C.W. Chu, M.J. Goldberg: J. Appl. Phys. 75, 1410 (1994)

9 H.C. Liou, R. Willacke, P.S. Ho: Thin Solid Films 323, 203 (1998)

10 G.D. Shyu, A.I. Isayev, C.T. Li: J. Polym. Sci. 39, 2552 (2001)

11 M. Ree, T.J. Shin, Y.H. Park, S.L. Kim, S.H. Woo, C.K. Cho, C.E. Park: J. Polym. Sci. Part B 36, 1261 (1998)

12 T. Liu, R. Samuels: J. Polym. Sci. Part B 39, 2481 (2001)

13 R. Ulrich, R. Torge: Appl. Opt. 12, 2901 (1973)

14 P.K. Tien: Appl. Opt. 10, 2395 (1971)

15 P.K. Tien, R. Ulrich: J. Opt. Soc. Am. 60, 1325 (1970)

16 P.H. Chantome, L. Escoubas, F. Flory: Appl. Opt. 41, 3127 (2002)

17 L. Levi: Applied Optics, Vol. 2 (Wiley, New York 1980)

18 R.D. Andrews, J.F. Rudd: J. Appl. Phys. 28, 1091 (1957)

19 D.L. Lee: Electromagnetic Principles of Integrated Optics (Wiley, New York 1986)

20 S. Monneret, P.H. Chantome, F. Flory: J. Opt. A: Pure Appl. Opt. 2, 188 (2000)

21 J. Brandrup, H.H. Immergut (Eds.): Polymer Handbook, 3rd edn. (Wiley, New York 1989)

22 J. Massaneda, F. Flory, E. Pelletier: Appl. Opt. 38, 4177 (1999)

23 C.P. Pollock: Fundamentals of Optoelectronics (Irwin, Chicago 1995)

24 F. Ay, S. Agan, A. Kocabas, C. Kocabas, A. Aydinli: submitted to Thin Solid Films (2003)

25 L.A. Hornak: Polymers for Lightwave and Integrated Optics (Marcel Dekker, New York 1992) 